\title{
TRIANGULAR ALGEBRAS AND IDEALS OF NEST ALGEBRAS
}

\author{
JOHN LINDSAY ORR
}

\section{INTRODUCTION}

Let $\mathscr{H}$ be a separable Hilbert space and $\mathscr{T} \subseteq \mathscr{B}(\mathscr{H})$ be an algebra of bounded operators. Say $\mathscr{T}$ is triangular if $\mathscr{T} \cap \mathscr{T}^{*}$ is a maximal abelian self-adjoint subalgebra (m.a.s.a.) of $\mathscr{B}(\mathscr{H})$ and call this m.a.s.a. the diagonal of $\mathscr{T}$. A triangular algebra is maximal triangular if it is not properly contained in any triangular algebra. Triangular algebras of operators have been studied for 30 years now, since the seminal paper of Kadison and Singer [6]. In this, they proposed the maximal triangular algebras as infinitedimensional generalizations of the upper triangular matrices and as the non-self-adjoint analogues of the von Neumann algebras. However, general questions on maximal triangular algebras have proved highly intractible and a theory of non-self-adjoint algebras based on these algebras has not developed. Nevertheless, special classes of triangular algebras have provided much of the motivation in the subsequent study of non-self-adjoint algebras, in the areas of nest algebras and CSL algebras (see [3]), algebras connected with ergodic actions [1] and non-self-adjoint subalgebras of certain $C^{*}$-algebras and von Neumann algebras $[10,11]$. Specifically, the study of nest algebras is now well developed and, as we show here, it is now possible to use the techniques of this subject to answer some of the hard questions which arose early in the study of triangular algebras.

In particular, it was hoped that, just as Murray and von Neumann essentially reduced the study of von Neumann algebras to the study of factors, analogous "primitive" maximal triangular

Received by the editors August 2, 1989 and, in revised form, May 24, 1990.

1980 Mathematics Subject Classification (1985 Revision). Primary 47D25, 47C05.

Key words and phrases. Triangular algebra, maximal triangular algebra, nest algebra, diagonal-disjoint ideal.

This research supported by an SERC(UK) Advanced Studentship. 
algebras might be identified. Ultimately this simplification in the case of von Neumann algebras rests on the regular behavior of a von Neumann algebra and its commutant under compression to the range of a projection in the center. Thus, the lack of answers to the following questions, first raised by Kadison and Singer and repeated by Erdos in [5], has been an obstacle to the development of a structural theory of maximal triangular algebras.

Let $\mathscr{T}$ be a maximal triangular algebra and $P$ a projection in its core (resp. diagonal). Is the compression of $\mathscr{T}$ to the range of $P$ always maximal triangular?

(The core of a triangular algebra is the von Neumann algebra generated by the projections onto its invariant subspaces.) We shall answer both of these questions in the negative. Since the core of a triangular algebra is contained in its diagonal, it will suffice to construct a counterexample for the question for the core.

Another obstacle to a deeper understanding of maximal triangular algebras is the dearth of concrete examples. Although maximal triangular algebras very unlike nest algebras were known to exist, the example (1) used in $\S 2$ below is the first concrete description of a maximal triangular algebra which is not a nest algebra. In fact, this example is a special case of a construction based on tensor products of nest algebras first suggested in a privately circulated addendum to [6] and described in $\S 3$ below. Potentially this construction yields many new triangular algebras. However, nothing could be said about any such algebras other than those which are nest algebras without some knowledge about the maximal diagonal-disjoint ideals of general nest algebras. (Heuristically, these are ideals of "strictly upper triangular" elements of the algebra; see $\S 2$ for the definition.) In $\S 2$ we see that every nest algebra has a unique maximum diagonal-disjoint ideal. This allows us to answer our main question above and, in $\S 3$, to describe many maximal triangular algebras containing tensor products of nest algebras. In $\S 4$ we apply the techniques developed to study the structure of nest algebras with continuous nest. We describe the class of ideals which is generated by the maximal ideals and we give a connectedness result which is related to the connectivity problem for the group of invertibles of a nest algebra.

A fuller discussion of these and other related results will appear elsewhere. I would like to express my deep gratitude to 
Dr. J. A. Erdos for his constant advice and encouragement throughout this work.

\section{Diagonal-Disjoint ideals}

A nest is a set of projections on $\mathscr{H}$ which is totally ordered under the comparison of ranges, contains 0 and $I$ (the identity operator) and is closed in the strong operator topology. If $\mathscr{N}$ is a nest, then the nest algebra, $\operatorname{Alg} \mathscr{N}$ is the set of bounded operators on $\mathscr{H}$ leaving invariant the ranges of the projections of $\mathscr{N}$. If the commutant, $\mathscr{N}^{\prime}$, of $\mathscr{N}$ is a m.a.s.a., then $\operatorname{Alg} \mathscr{N}$ is triangular and we say that $\mathscr{N}$ is multiplicity-free. A nest $\mathscr{N}$ is continuous if it is order isomorphic to the interval, $[0,1]$. A two-sided ideal, $\mathscr{I}$, of $\operatorname{Alg} \mathscr{N}$ is said to be diagonal-disjoint if $\mathscr{I} \cap \mathcal{N}^{\prime}=\{0\}$. Let $\mathscr{N}$ be a multiplicity-free nest. We connect with triangular algebras by observing that the set of operators on $\mathscr{H} \oplus \mathscr{H}$ given by

$$
\left(\begin{array}{cc}
\operatorname{Alg} \mathscr{N} & \operatorname{Alg} \mathscr{N} \\
\mathscr{J} & \operatorname{Alg} \mathscr{N}
\end{array}\right),
$$

where $\mathscr{I}$ is a diagonal-disjoint ideal, is a triangular algebra, and if $\mathscr{I}$ is maximal as a diagonal-disjoint ideal then the algebra is maximal triangular. We now identify the largest diagonal-disjoint ideal of $\operatorname{Alg} \mathscr{N}$. Let $\mathscr{N}$ be a nest and let $\mathscr{R}_{\mathcal{N}}^{\infty}$ be the set of operators $X$ in $\operatorname{Alg} \mathscr{N}$ such that, for each $\varepsilon>0$, there is a collection of pairwise orthogonal projections, $E_{\alpha}=G_{\alpha}-F_{\alpha}$ where $G_{\alpha}, F_{\alpha}$ are in $\mathscr{N}$, such that $\left\|E_{\alpha} X E_{\alpha}\right\| \leq \varepsilon$ for all $\alpha . \mathscr{R}_{\mathscr{N}}^{\infty}$ is a diagonaldisjoint ideal of $\operatorname{Alg} \mathscr{N}$ which was introduced by Larson in [8]. Our main result on diagonal-disjoint ideals is the following:

Theorem 2.1. For any nest $\mathscr{N}, \mathscr{R}_{\mathcal{N}}^{\infty}$ is the largest diagonal-disjoint ideal of $\operatorname{Alg} \mathscr{N}$.

The major difficulties in the theorem are present when we consider $\mathscr{N}$ a continuous nest. We may parameterize $\mathscr{N}$ as $\left\{N_{t}: t \in\right.$ $[0,1]\}$ in such a way that the induced projection-valued LebesgueStieltjes measure on $[0,1]$ is mutually absolutely continuous with Lebesgue measure [4]. For each $X$ in $\operatorname{Alg} \mathscr{N}$ we define an upper semicontinuous function, $j_{X}$, on the interval $(0,1)$ (in the spirit of the functions defined by Ringrose [12]) by

$$
j_{X}(t)=\lim _{a \rightarrow 0+}\left\|\left(N_{t+a}-N_{t-a}\right) X\left(N_{t+a}-N_{t-a}\right)\right\| .
$$

Heuristically, this function should be thought of as measuring the size of $X$ "on the diagonal." The behavior of the function $j_{X}$ is sufficient to determine whether or not $X$ lies in $\mathscr{R}_{\mathscr{N}}^{\infty}$. 
Lemma 2.2. ${ }^{1}$ Let $\mathcal{N}$ be a continuous nest. Then

$$
\begin{array}{r}
\mathscr{R}_{\mathscr{N}}^{\infty}=\left\{X \in \operatorname{Alg} \mathscr{N}: j_{X}=0\right. \\
\text { almost everywhere in Lebesgue measure }\} .
\end{array}
$$

Conversely, if $j_{X}$ is nonzero on a large (i.e. nonnull) set, we can interpolate from $X$ to a projection in the core of $\operatorname{Alg} \mathcal{N}$.

Theorem 2.3. Let $\mathscr{N}$ be a continuous nest, $X \in \operatorname{Alg} \mathscr{N}$ and $\eta>$ 0 . Let $\mathscr{E}$ be the Lebesgue-Stieltjes measure on $[0,1]$ such that $\mathscr{E}((a, b])=N_{b}-N_{a}$. Then, if $S=\left\{t: j_{X}(t) \geq \eta\right\}$, there are $A$, $B$ in $\operatorname{Alg} \mathscr{N}$ such that

$$
A X B=\mathscr{E}(\mathscr{S}) \in \mathscr{N}^{\prime \prime} .
$$

Theorem 2.3 is at the heart of this work and its proof makes frequent use of the deep Similarity Theorem for nests [2]. Briefly, we construct an idempotent, $Q$, which is algebraically equivalent to $\mathscr{E}(\mathscr{S})$ (using the recent work of Larson and Pitts [9]) but which is a sum of idempotents in $\mathscr{R}_{\mathscr{N}}^{\infty}$. Each of these idempotents and hence, as it happens, $Q$, can be factorized by shift-type operators and the problem is reduced to simultaneously interpolating these fairly concrete operators. When $\mathscr{N}$ is continuous, Theorem 2.1 is then immediate from Theorem 2.3 and Lemma 2.2.

We now return to our question on triangular algebras. Let $\mathscr{N}$ be continuous and multiplicity-free. For any projection, $F \in \mathscr{N}^{\prime}$, note that $F \mathscr{N}=\left\{\left.F N\right|_{F \mathscr{H}}: N \in \mathscr{N}\right\}$ is also a continuous nest on $F \mathscr{H}$. We need a technical lemma.

Lemma 2.4. Let $\mathscr{N}$ be a continuous nest. Then there is a projection, $F$, in $\mathscr{N}^{\prime \prime}$ such that

$$
\left.F \mathscr{R}_{\mathscr{N}}^{\infty}\right|_{F \mathscr{H}} \varsubsetneqq \mathscr{R}_{(F \mathcal{N})}^{\infty} \text {. }
$$

Recalling the construction (1) of a triangular algebra, we take $\mathscr{I}=\mathscr{R}_{\mathcal{N}}^{\infty}$ and note that the projection, $\left(\begin{array}{ll}F & 0 \\ 0 & F\end{array}\right)$ (where $F$ is as in the last lemma), is in the core of this maximal triangular algebra and that the compression to its range is equal to,

$$
\left(\begin{array}{cc}
\operatorname{Alg} F \mathscr{N} & \operatorname{Alg} F \mathscr{N} \\
\left.F \mathscr{R}_{\mathscr{N}}^{\infty}\right|_{F \mathscr{H}} & \operatorname{Alg} F \mathscr{N}
\end{array}\right)
$$

This is properly contained in a maximal triangular algebra of form (1), with entries form $\operatorname{Alg} F \mathscr{N}$ and $\mathscr{R}_{F \mathcal{N}}^{\infty}$, and we are finished.

${ }^{1}$ This has been independently observed by X. Dai (personal communication). 


\section{TENSOR PRODUCT CONSTRUCTIONS}

If $\mathscr{T}_{1}, \mathscr{T}_{2}$ are triangular nest algebras, then their spatial tensor product is triangular [7]. In the case that $\mathscr{T}_{2}$ is the algebra of $2 \times 2$ upper triangular matrices, Theorem 2.1 shows that example (1) is a maximal triangular algebra containing $\mathscr{T}_{1} \otimes \mathscr{T}_{2}$. In an unpublished final chapter to [6], Kadison and Singer proposed a construction for maximal triangular algebras, by taking tensor products of triangular nest algebras and enlarging by "lower triangular terms" coming from a maximal diagonal-disjoint ideal. We can realize this program as follows:

Theorem 3.1. Let $\operatorname{Alg} \mathscr{N}_{1}$ and $\operatorname{Alg} \mathscr{N}_{2}$ be triangular nest algebras acting on spaces, $\mathscr{H}_{1}, \mathscr{H}_{2}$, respectively. Then there is a unique maximal triangular algebra, $\mathscr{T}$, satisfying

$$
\operatorname{Alg} \mathscr{N}_{1} \otimes \operatorname{Alg} \mathscr{N}_{2} \subseteq \mathscr{T} \subseteq \operatorname{Alg} \mathscr{N}_{1} \otimes \mathscr{B}\left(\mathscr{H}_{2}\right) .
$$

Furthermore, $\mathscr{T}$ admits a concrete description. The set of elementary tensors, $N_{1} \otimes I_{2}$ (for $N_{1} \in \mathscr{N}_{1}$ ), is a nest, written $\mathscr{N}_{1} \otimes I_{2}$, and $\mathscr{T}$ is the set of all operators $X \in \operatorname{Alg} \mathscr{N}_{1} \otimes \mathscr{B}\left(\mathscr{H}_{2}\right)$ such that (i) whenever $N_{2} \in \mathscr{N}_{2}$ has both an immediate predecessor and successor in $\mathscr{N}_{2}$, then

$$
I_{1} \otimes N_{2}^{\perp} X I_{1} \otimes N_{2} \in \mathscr{R}_{\mathscr{N}_{1} \otimes I_{2}}^{\infty}
$$

and (ii) whenever $N_{2}>N_{2}^{\prime}>N_{2}^{\prime \prime}$ are in $\mathscr{N}_{2}$, then

$$
I_{1} \otimes N_{2}^{\perp} X I_{1} \otimes N_{2}^{\prime \prime} \in \mathscr{R}_{\mathscr{N}_{1} \otimes I_{2}}^{\infty} .
$$

This construction and its extensions yield many new maximal triangular algebras related to, but rather different from, nest algebras. Further properties of these algebras will be discussed elsewhere.

\section{IDEALS AND CONNECTEDNESS}

Let $\mathscr{N}$ be continuous but not necessarily multiplicity free. We define an ideal in analogy to the description of $\mathscr{R}_{\mathcal{N}}^{\infty}$ in Lemma 2.2. Define $\mathscr{I}_{\mathscr{N}}^{\infty}$ to be the set of operators, $\left\{X \in \operatorname{Alg} \mathscr{N}: j_{X}(t)\right.$ vanishes outside a meager set $\}$. There is a correspondence between ideals of $\operatorname{Alg} \mathscr{N}$ which contain $\mathscr{I}_{\mathcal{N}}^{\infty}$ and certain families of upper semicontinuous functions on the interval $(0,1)$.

Theorem 4.1. Let $\mathscr{C}$ be a uniformly closed hereditary cone (under pointwise ordering) of positive-valued upper semicontinuous functions on $(0,1)$ which contains the characteristic functions of all the closed sets with empty interior. Then $\left\{X \in \operatorname{Alg} \mathscr{N}: j_{X} \in \mathscr{C}\right\}$ 
is a norm-closed, two-sided ideal of $\operatorname{Alg} \mathscr{N}$ containing $\mathscr{I}_{\mathcal{N}}^{\infty}$. Furthermore, every such ideal arises in this way and the maps,

$$
\begin{aligned}
\mathscr{C} & \mapsto\left\{X \in \operatorname{Alg} \mathscr{N}: j_{X} \in \mathscr{C}\right\} \\
\mathscr{I} & \mapsto\left\{j_{X}: X \in \mathscr{I}\right\}
\end{aligned}
$$

are mutual inverses.

Corollary 4.2. Every closed ideal of $\operatorname{Alg} \mathscr{N}$ which contains $\mathscr{I}_{\mathcal{N}}^{\infty}$ is equal to an intersection of maximal two-sided ideals. In particular, $\mathcal{I}_{\mathcal{N}}^{\infty}$ is equal to the intersection of all the maximal two-sided ideals of $\operatorname{Alg} \mathscr{N}$ (i.e. it is the strong radical of $\operatorname{Alg} \mathscr{N}$ ).

It is a longstanding and intractible problem whether the invertible elements of a nest algebra are path-connected. Because Theorem 2.3 gives a sharp condition on $X$ for the existence of operators $A, B$ such that $A X B=I$ in the algebra of a continuous nest, we can prove the following analogous result.

Theorem 4.3. Let $\mathcal{N}$ be a continuous nest. Then the set of operators $X$ in $\operatorname{Alg} \mathcal{N}$ for which there exist $A, B \in \operatorname{Alg} \mathscr{N}$ with $A X B=I$ is path-connected by norm continuous paths.

In particular, every invertible element of $\operatorname{Alg} \mathscr{N}$ is connected to the identity by a path which never intersects a proper ideal of $\operatorname{Alg} \mathscr{N}$.

\section{REFERENCES}

1. W. B. Arveson, Operator algebras and measure-preserving automorphisms, Acta Math. 118 (1967), 95-109.

2. K. R. Davidson, Similarity and compact perturbations of nest algebras, J. Reine Angew. Math. 348 (1984), 286-294.

3. __ Nest algebras, Research notes in Math., vol. 191, Longman Scientific and Technical, Essex, 1988.

4. J. A. Erdos, Unitary invariants for nests, Pacific J. Math. 23 (1967), 229256.

5. __ Some questions concerning triangular operator algebras, Proc. Royal Irish Acad. 74A (1974), 223-232.

6. R. V. Kadison and I. M. Singer, Triangular operator algebras, Amer. J. Math. 82 (1960), 227-259.

7. J. Kraus, Tensor products of reflexive algebras, J. London Math. Soc. 28 (1983), 350-358.

8. D. R. Larson, Nest algebras and similarity transformations, Ann. of Math. (2) 121 (1985), 409-427.

9. D. R. Larson and D. Pitts, Idempotents in nest algebras, J. Funct. Anal. (to appear). 
10. P. S. Muhly, K.-S. Saito, and B. Solel, Coordinates for triangular operator algebras, Ann. of Math. (2) 127 (1988), 245-278.

11. P. S. Muhly and B. Solel, Subalgebras of groupoid $C^{*}$-algebras, preprint, 1988.

12. J. R. Ringrose, On some algebras of operators, Proc. London Math. Soc. 15 (1965), 61-83.

Department of Mathematics, King's College, Strand, London, EngLAND

Current address: Department of Pure Mathematics, University of Waterloo, Waterloo, Ontario, Canada

E-mail address: JORR @WATSERV1.uWATERLOO.CA 
